\title{
The role of prenatal exposures on body fat patterns at 7 years: Intrauterine programming or birthweight effects?
}

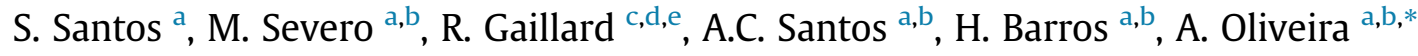 \\ ${ }^{a}$ EPI-Unit, Institute of Public Health, University of Porto, Porto, Portugal \\ ${ }^{b}$ Department of Clinical Epidemiology, Predictive Medicine and Public Health, University of Porto Medical School, Porto, Portugal \\ ${ }^{c}$ The Generation R Study Group, Erasmus MC, University Medical Center, Rotterdam, The Netherlands \\ ${ }^{d}$ Department of Pediatrics, Erasmus MC, University Medical Center, Rotterdam, The Netherlands \\ ${ }^{e}$ Department of Epidemiology, Erasmus MC, University Medical Center, Rotterdam, The Netherlands
}

Received 23 March 2016; received in revised form 3 June 2016; accepted 21 June 2016

Available online 28 June 2016

\section{KEYWORDS \\ Fetal programming; \\ Birth weight; \\ Body fat; \\ Path analysis; \\ Children; \\ Cohort studies}

\begin{abstract}
Background and aims: It remains unknown whether the effects of prenatal exposures on child's adiposity reflect entirely intrauterine programming. We aimed to assess the effects of maternal gestational weight gain, diabetes and smoking on the child's body fat patterns, disentangling the direct (through intrauterine programming) and indirect (through birthweight) effects. Methods and results: We included 4747 singleton 7-year-old children from the Generation XXI birth cohort (Porto, Portugal). At birth, maternal and newborn's characteristics were obtained. Anthropometrics were measured at age 7 years and body fat patterns were identified by principal component analysis. Path analysis was used to quantify direct, indirect and total effects of gestational weight gain, diabetes and smoking on body fat patterns. Pattern 1 was characterized by strong factor loadings with body mass index, fat mass index and waist-to-height ratio (fat quantity) and pattern 2 with waist-to-hip ratio, waist-to-thigh ratio, and waist-to-weight ratio (fat distribution). The positive total effect of maternal gestational weight gain and diabetes on the child's fat quantity was mainly through a direct pathway, responsible for $91.7 \%$ and $83.7 \%$ of total effects, respectively ( $\beta=0.022 ; 95 \%$ Confidence Interval $(\mathrm{CI}): 0.017,0.027 ; \beta=0.041 ; 95 \% \mathrm{CI}:-0.011$, 0.093). No effects on fat distribution were found. Maternal prenatal smoking had a positive direct effect on patterns 1 and 2, explaining $94.9 \%$ and $76.1 \%$ of total effects, respectively.

Conclusion: The effects of maternal gestational weight gain, diabetes and smoking on a child's fat quantity seem to be mainly through intrauterine programming. Maternal smoking also showed a positive direct effect on child's fat distribution.

๑) 2016 The Italian Society of Diabetology, the Italian Society for the Study of Atherosclerosis, the Italian Society of Human Nutrition, and the Department of Clinical Medicine and Surgery, Federico II University. Published by Elsevier B.V. All rights reserved.
\end{abstract}

\section{Introduction}

Intrauterine programming has been pointed out, along with genetic predisposition, as a major cause of childhood

\footnotetext{
* Corresponding author. Institute of Public Health, University of Porto, Rua das Taipas 135, 4050-600 Porto, Portugal. Tel.: +351 222061820; fax: +351222061821 .

E-mail address: acmatos@med.up.pt (A. Oliveira).
}

obesity [1]. A large body of evidence has focused on adiposity programming by maternal under- or overnutrition, weight gain, diabetes and smoking during pregnancy [2]. These exposures are likely to affect the development of adipocytes and their capacity to expand or contract, the appetite control system and the energy metabolism in later life [3]. However, it remains unknown whether the observed effects in these previous studies reflect entirely intrauterine programming. Confounding by 
lifestyles or genetics and the possibility of pathways mediated by other exposures might still be an issue.

Previous studies have addressed the effect of prenatal exposures on the risk of childhood obesity with and without adjustment for birthweight [4-7]. Since birthweight may be a mediator in these associations, the adjustment for birthweight might be inappropriate. First, the effect estimate obtained from a model adjusted for birth weight does no longer correspond to the total effect. Secondly, the approach of simply adjusting for mediators in the regression models is prone to bias and may produce flawed conclusions [8]. To our knowledge, no study has discussed separately the intrauterine programming effects from those mediated by birthweight. Assessing these effects separately will help to get further insight into the paths and mechanisms involved and their specific contributions to the associations between prenatal exposures and adiposity in later life.

Most studies looking at the relation between prenatal exposures and later adiposity have used proxies and have relied on single measures for defining adiposity, which might have limited their ability to detect associations [9]. The combination of anthropometric indices according to their inter-correlations into robust and uncorrelated patterns of body fat could be a more accurate and yet simple approach of evaluating childhood adiposity.

The present study aimed to assess the effects of prenatal exposures (gestational weight gain, diabetes and smoking) on body fat patterns of 7-year-old children. A path analysis helped to understand whether these effects are mainly explained by a direct (through intrauterine programming) or indirect pathway (through birthweight).

\section{Methods}

\section{Study population}

This study included participants from Generation XXI - a population-based birth cohort assembled during 2005-2006 at all public maternity units of Porto, Portugal. Of the invited mothers, 91.4\% accepted to participate, corresponding to 8647 newborns. A detailed description of the cohort methodology was previously reported [10]. At the age of 7 years (2012-2014), 6889 children were reevaluated (79.7\% of the entire cohort), of which 5849 provided data by face-to-face interviews.

For the definition of body fat patterns, of 5849 children who attended the face-to-face interviews at 7 years old, we excluded 130 children with missing information on anthropometrics and/or tetra-polar bioelectric impedance, yielding a total of 5719 children (48.5\% females). Further, we excluded 212 twins and 760 children who lacked data on selected variables of interest. The final study sample included 4747 children ( $48.6 \%$ females). The comparison between participants $(\mathrm{n}=4747)$ and non-participants (excluding twins, $\mathrm{n}=3604$ ) showed that mothers in this study were slightly older [mean (standard deviation, $\mathrm{SD})=29.6$ (5.30) vs. 28.1 (5.94) years old] and higher educated [mean (SD) $=11.1$ (4.27) vs. 9.5 (4.04) years] than mothers who were not included in this study. However, the magnitude of the differences was not high (Cohen's effect size values [11] of 0.27 and 0.38 for maternal age and education, respectively), suggesting that the differences were mostly due to the large sample size rather than due to large differences between participants' characteristics.

\section{Data collection}

\section{Baseline evaluation}

Data on maternal characteristics were collected in face-toface interviews, within $72 \mathrm{~h}$ after delivery, during the hospital stay. Maternal age and educational level at birth were recorded as completed years of aging and schooling. Information on prenatal smoking habits was collected and mothers were grouped into never or ever smokers during pregnancy. Pre-pregnancy weight was obtained through recall. Maternal height was measured by interviewers, and, when not possible, was abstracted from the national identity card. Pre-pregnancy body mass index $\left(\mathrm{kg} / \mathrm{m}^{2}\right.$, BMI) was calculated. Maternal gestational weight gain was calculated as the difference between the final pre-delivery weight, reported by mothers, and the pre-pregnancy weight. Personal history of diabetes mellitus was considered present when participants reported a medical diagnosis of this condition before the current pregnancy. Gestational diabetes was considered present when recorded on obstetrical records as a diagnosis during the current pregnancy. Mothers were grouped into never or ever had a diagnosis of type 1 or type 2 diabetes mellitus or gestational diabetes. Clinical records were reviewed at birth to retrieve data on gestational age and birthweight. Gestational age was estimated by ultrasound and, when it was missing, it was estimated based on the last menstrual period. The z-scores of birthweight for gestational age were calculated according to a sex-specific, populationbased Canadian reference [12].

\section{Re-evaluation at 7 years old}

Anthropometrics were obtained by trained personnel with children in underwear and barefoot, according to standard procedures. Body weight and height were measured using a digital scale $\left(\right.$ TANITA $^{\circledR}$ ) and a wall stadiometer $\left(\right.$ SECA $\left.^{\circledR}\right)$, respectively. Waist circumference was measured at the umbilicus level, with abdomen relaxed and hip circumference was measured at the level of the greatest posterior protuberance of the buttocks. Thigh circumference was measured at the position around the mid-thigh and perpendicular to the long axis of the thigh, with the leg slightly flexed. All anthropometrics were measured to the nearest $0.1 \mathrm{~kg}$ or $\mathrm{cm}$. Body mass index was calculated ( $\mathrm{kg} /$ $\mathrm{m}^{2}$ ). The waist-to-height, waist-to-hip and waist-to-thigh ratios were calculated as waist circumference divided by height, hip circumference and thigh circumference, respectively. The relationship between waist circumference and weight was assessed using a log-log regression analysis. Log-waist circumference was regressed on log-weight. The gradient of the regression line was approximately 0.5 , 
corresponding to the value by which weight should be raised in order to calculate a measure uncorrelated with it. Waist-to-weight ratio was calculated as waist circumference divided by the square root of weight.

Bioelectric impedance analysis was performed using a tetra-polar device (BIA 101 Anniversary, Akern, Florence, Italy). Four surface electrodes were placed on the right wrist, ankle, hand and foot with the child lying horizontally. Measurements were performed at least $30 \mathrm{~min}$ after the last meal. Fat free mass was determined using Schaefer et al. equation [13] and fat mass was derived accordingly. Fat mass was divided by the squared height to obtain the fat mass index [14], in which fat mass was effectively uncorrelated with height, as confirmed by a log-log regression analysis.

\section{Ethics}

All phases of the study complied with the Ethical Principles for Medical Research Involving Human Subjects expressed in the Declaration of Helsinki. The study was approved by the University of Porto Medical School/S. João Hospital Centre ethics committee and a signed informed consent according Helsinki was required for all participants.

\section{Statistical analysis}

Principal component analysis was applied to BMI, waistto-height ratio, waist-to-hip ratio, waist-to-thigh ratio, waist-to-weight ratio, and fat mass index to identify uncorrelated patterns of body fat. The number of factors was decided using the Kaiser's criterion, by which factors with an eigenvalue $\geq 1.0$ are retained. Varimax rotation was performed to simplify the interpretation of the factor loadings structure. The interpretation of the factors was based on those measures with factor loadings higher than 0.30 , considering $0.30-0.70$ as moderate and $\geq 0.70$ as strong factor loadings. The scores that were entered in all subsequent analyses were calculated using the regression method with standardized scores. Analyses were conducted using the $\mathrm{R}^{\circledR}$ software version 3.0.1.

Path analysis determines whether data fit well within a prespecified causal model and allows the study of direct and indirect effects with multiple independent and dependent variables [15]. Path analysis was used to quantify direct, indirect (by multiplication of path regression coefficients involved) and total effects (by summing direct and indirect effects) of prenatal exposures on body fat patterns, taking into consideration the relationships depicted in the causal diagram presented as Fig. 1. The path analysis used linear regression models for continuous outcomes and probit regression models for categorical outcomes and results were presented as regression coefficients ( $\beta$ ) and corresponding $95 \%$ confidence intervals $(95 \% \mathrm{CI})$. The adjustment sets for each regression model are presented in the footnotes of Fig. 1. Covariates were included in the analyses when they changed the effect estimates substantially ( $>10 \%$ ): maternal pre-pregnancy body mass index, age and educational level at birth. Other covariates such as parity, mode of delivery, child's
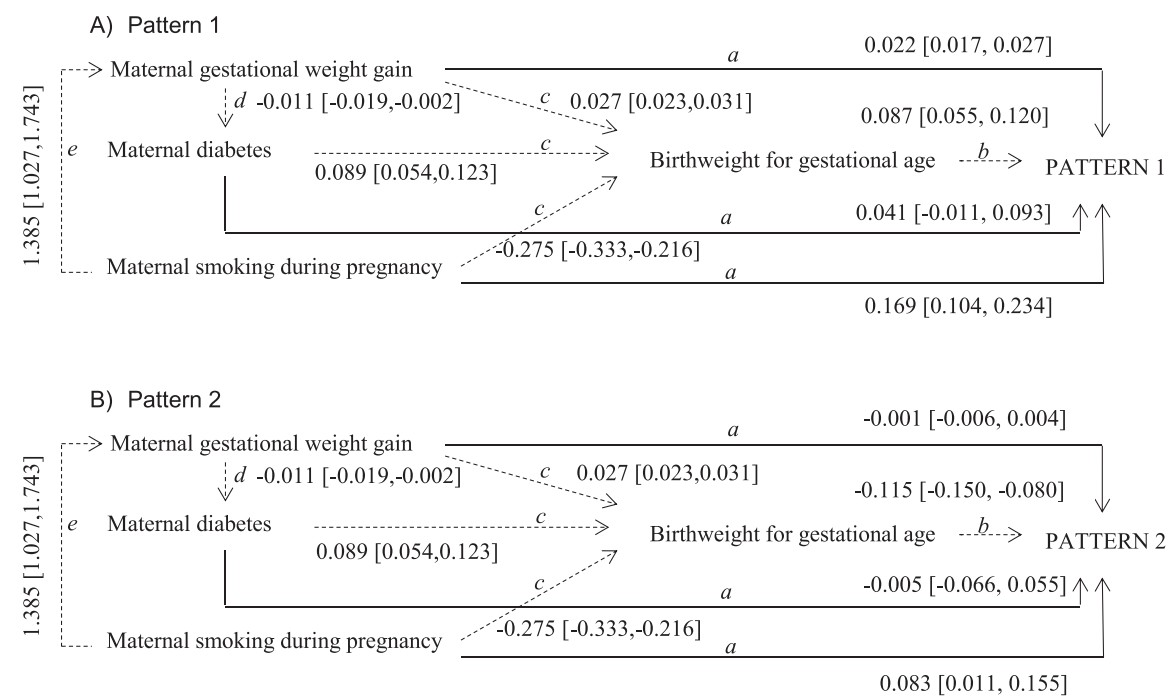

Figure 1 Causal diagram for the effects of prenatal exposures on body fat patterns identified by principal component analysis at 7-year-old children ${ }^{1,2}$.

${ }^{1}$ Direct effects correspond to the intrauterine programming effects (solid arrows) and the indirect effects correspond to the effects mediated by other exposures (dashed arrows).

${ }^{2}$ Adjustment sets for each regression model:

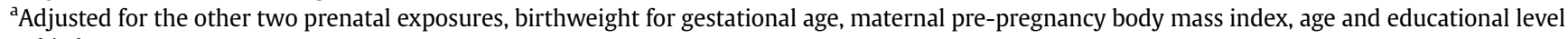
at birth.

${ }^{\mathrm{b}}$ Adjusted for the prenatal exposures, maternal pre-pregnancy body mass index, age and educational level at birth.

${ }^{\mathrm{c} A d j u s t e d ~ f o r ~ t h e ~ o t h e r ~ t w o ~ p r e n a t a l ~ e x p o s u r e s, ~ a n d ~ m a t e r n a l ~ p r e-p r e g n a n c y ~ b o d y ~ m a s s ~ i n d e x . ~}{ }^{\mathrm{d} A d j u s t e d ~ f o r ~ m a t e r n a l ~ p r e-p r e g n a n c y ~ b o d y ~ m a s s ~}$ index and age at birth.

${ }^{\mathrm{e}}$ Adjusted for maternal pre-pregnancy body mass index, age and educational level at birth. 
sex, breastfeeding duration, physical exercise and fruit/ vegetables intake were tested but did not fulfill the criterion for being considered confounders and therefore were not included in the regression models. Models were fitted, simultaneously for all body fat patterns identified, with Mplus software version 5.2 (Muthén \& Muthén, Los Angeles, CA, USA). A Comparative Fit Index (CFI) and a Tucker-Lewis Index (TLI) $\geq 0.90$ and a Root Mean Square Error of Approximation (RMSEA) close to zero were used as criteria to support the goodness of fit [16]. No significant interactions between prenatal exposures and child's sex in the associations with body fat patterns were found.

\section{Results}

Characteristics of participants are shown in Table 1. Two body fat patterns, similar for both sexes, were identified: a pattern 1 characterized by strong factor loadings with BMI, fat mass index and waist-to-height ratio (fat quantity) and a pattern 2 characterized by strong factor loadings with waist-to-hip ratio, waist-to-thigh ratio and waist-toweight ratio (fat distribution) (Table 2). Both patterns

Table 1 Characteristics of study participants ( $n=4747){ }^{\mathrm{a}}$

\begin{tabular}{ll}
\hline Child's characteristics & Mean (SD) \\
\hline Birthweight for gestational age, z-scores & $-0.3(0.85)$ \\
Body mass index at $7 \mathrm{y}, \mathrm{kg} / \mathrm{m}^{2}$ & $17.1(2.51)$ \\
Fat mass index at $7 \mathrm{y}, \mathrm{kg} / \mathrm{m}^{2}$ & $3.2(2.33)$ \\
Waist-to-height ratio at $7 \mathrm{y}$ & $0.5(0.05)$ \\
Waist-to-hip ratio at $7 \mathrm{y}$ & $0.9(0.05)$ \\
Waist-to-thigh ratio at $7 \mathrm{y}$ & $1.6(0.10)$ \\
Waist-to-weight ratio at $7 \mathrm{y}, \mathrm{cm} / \sqrt{ } \mathrm{kg}$ & $10.2(0.48)$ \\
Maternal characteristics & \\
\hline Age at birth, years & $29.6(5.30)$ \\
Educational level at birth, years & $11.1(4.27)$ \\
Pre-pregnancy body mass index, kg/m ${ }^{2}$ & $23.8(4.16)$ \\
Gestational weight gain, kg & $13.7(5.77)$ \\
& $\mathbf{n}(\%)$ \\
Diabetes (type 1 or type 2 diabetes & $327(6.9)$ \\
$\quad$ mellitus or gestational diabetes) & \\
Smokers during pregnancy & $992(20.9)$ \\
\hline a SD, standard deviation. &
\end{tabular}

Table 2 Factor loadings, obtained from principal component analysis, for anthropometric and bioelectric impedance measures $(\mathrm{n}=5719)$.

\begin{tabular}{lll}
\hline & \multicolumn{2}{l}{ Factor loadings } \\
\cline { 2 - 3 } & Pattern $1^{\mathrm{a}}$ & Pattern $2^{\mathrm{b}}$ \\
\hline Body mass index, $\mathrm{kg} / \mathrm{m}^{2}$ & 0.980 & 0.051 \\
Fat mass index, $\mathrm{kg} / \mathrm{m}^{2}$ & 0.937 & 0.059 \\
Waist-to-height ratio & 0.873 & 0.454 \\
Waist-to-hip ratio & 0.277 & 0.877 \\
Waist-to-thigh ratio & -0.044 & 0.893 \\
Waist-to-weight ratio, cm $/ \sqrt{ } \mathrm{kg}$ & 0.202 & 0.895 \\
Variance explained & $45.3 \%$ & $43.0 \%$ \\
Cumulative variance explained & $45.3 \%$ & $88.3 \%$ \\
\hline a Representing fat quantity. & & \\
b Representing fat distribution. & & \\
\end{tabular}

explained $88.3 \%$ of the total variance ( $45.3 \%$ was explained by pattern 1 and $43.0 \%$ by pattern 2 ).

Fig. 1 shows the theoretical causal diagram for the effects of prenatal exposures on body fat patterns, in which the direct effects correspond to the programming effects and the indirect effects correspond to the effects mediated by other exposures. Fig. 1 also shows the effect estimates for each path. Table 3 presents the estimates of direct, indirect and total effects of prenatal exposures on the body fat patterns. The overall fit of the model was good: $\mathrm{CFI}=0.993$, TLI $=0.954$ and RMSEA $=0.023$. Maternal gestational weight gain had a positive total effect on pattern 1 ( $\beta=0.024 ; 95 \% \mathrm{CI}: 0.019,0.028)$, mainly due to a direct pathway that was responsible for $91.7 \%$ of the total effect ( $\beta=0.022 ; 95 \% \mathrm{CI}: 0.017,0.027)$. We observed a positive total effect of borderline statistical significance of maternal diabetes on pattern 1 ( $\beta=0.049$; 95\% CI: $-0.003,0.101)$, mainly due to a direct pathway that was responsible for $83.7 \%$ of the total effect $(\beta=0.041 ; 95 \% \mathrm{CI}$ : $-0.011,0.093)$. The indirect effects through birthweight of these prenatal exposures on both patterns were statistically significant but close to 0 . After excluding the mothers with type 1 and type 2 diabetes mellitus from the analyses, similar results were observed for the associations of maternal gestational diabetes with both body fat patterns at 7 years old (results not shown). Maternal smoking during pregnancy had a positive total effect on both body fat patterns (pattern 1: $\beta=0.178$; 95\% CI: 0.113, 0.243; pattern $2: \beta=0.109 ; 95 \% \mathrm{CI}: 0.038,0.180)$, mostly due to a direct effect that explained $94.9 \%$ and $76.1 \%$ of the total effect on patterns 1 and 2, respectively. A negative indirect effect through birthweight on pattern 1 ( $\beta=-0.024 ; 95 \%$ CI: $-0.034,-0.014)$ and a positive indirect effect through birthweight on pattern 2 ( $\beta=0.032 ; 95 \% \mathrm{CI}: 0.020,0.043$ ) were also found, i.e., prenatal smoking was negatively associated with birthweight $(\beta=-0.275$; $95 \% \mathrm{CI}:-0.333$, $-0.216)$ which was positively related to pattern 1 ( $\beta=0.087 ; 95 \% \mathrm{CI}: 0.055,0.120)$ and negatively related to pattern 2 ( $\beta=-0.115$; 95\% CI: $-0.150,-0.080$ ) (Table 3 and Fig. 1). We observed similar results in the principal component analysis and path analysis when we used the age- and sex-adjusted BMI standard deviation scores based on the World Health Organization Child Growth Standards (results not shown).

\section{Discussion}

The effects of maternal weight gain, diabetes and smoking during pregnancy on body fat quantity of 7-year-old children seem to be mainly through their intrauterine programing effects. These effects on child's adiposity prevailed over the indirect effects through birthweight. Maternal smoking during pregnancy also showed a positive direct effect on body fat distribution of children.

A previous meta-analysis showed that offspring of women with excessive gestational weight gain were at an increased risk of obesity, compared with offspring of women with adequate gestational weight gain [6]. Our study showed that this effect of maternal gestational 
Table 3 Estimates of direct, indirect and total effects of prenatal exposures on body fat patterns of 7-year-old children, calculated by path analysis $(\mathrm{n}=4747)^{\text {a }}$

\begin{tabular}{|c|c|c|c|c|}
\hline & \multicolumn{2}{|l|}{ Pattern 1} & \multicolumn{2}{|l|}{ Pattern 2} \\
\hline & $\beta[95 \% \mathrm{CI}]$ & $\%$ of total effect & $\beta[95 \% \mathrm{CI}]$ & $\%$ of total effect \\
\hline \multicolumn{5}{|c|}{ Maternal gestational weight gain } \\
\hline Direct effect & $0.022[0.017,0.027]$ & $91.7 \%$ & $-0.001[-0.006,0.004]$ & $25 \%$ \\
\hline Indirect effects & $0.002[0.001,0.003]$ & $8.3 \%$ & $-0.003[-0.004,-0.002]$ & $75 \%$ \\
\hline $\begin{array}{l}\text { Indirect effect through } \\
\text { birthweight }\end{array}$ & $0.002[0.001,0.003]$ & $100 \%$ & $-0.003[-0.004,-0.002]$ & $100 \%$ \\
\hline Total effect & $0.024[0.019,0.028]$ & & $-0.004[-0.009,0.001]$ & \\
\hline \multicolumn{5}{|l|}{ Maternal diabetes } \\
\hline Direct effect & $0.041[-0.011,0.093]$ & $83.7 \%$ & $-0.005[-0.066,0.055]$ & $33.3 \%$ \\
\hline Indirect effects & $0.008[0.004,0.012]$ & $16.3 \%$ & $-0.010[-0.015,-0.005]$ & $66.7 \%$ \\
\hline $\begin{array}{l}\text { Indirect effect through } \\
\text { birthweight }\end{array}$ & $0.008[0.004,0.012]$ & $100 \%{ }^{\mathrm{b}}$ & $-0.010[-0.015,-0.005]$ & $100 \%$ \\
\hline Total effect & $0.049[-0.003,0.101]$ & & $-0.015[-0.076,0.045]$ & \\
\hline \multicolumn{5}{|c|}{ Maternal smoking during pregnancy } \\
\hline Direct effect & $0.169[0.104,0.234]$ & $94.9 \%$ & $0.083[0.011,0.155]$ & $76.1 \%$ \\
\hline Indirect effects & $0.009[-0.006,0.024]$ & $5.1 \%$ & $0.026[0.012,0.040]$ & $23.9 \%$ \\
\hline $\begin{array}{l}\text { Indirect effect through } \\
\text { birthweight }\end{array}$ & $-0.024[-0.034,-0.014]$ & $-^{c}$ & $0.032[0.020,0.043]$ & $-{ }^{c}$ \\
\hline Total effect & $0.178[0.113,0.243]$ & & $0.109[0.038,0.180]$ & \\
\hline
\end{tabular}

weight gain on child's body fat quantity is mainly through intrauterine programming effects instead of indirect effects through birthweight. Higher maternal weight gain in pregnancy might program higher adiposity in the offspring through fetal over-nutrition that has been associated with a permanent increase in the capacity of adipocytes to store lipids [17], and with excessive appetite postnatally [18]. Previous studies found that higher maternal gestational weight gain was associated with a central fat distribution in the offspring, mainly assessed by waist circumference [19-21]. As waist circumference and BMI are strongly correlated [22], these associations could be reflecting the well-established positive effect of maternal gestational weight gain on fat quantity. In this study, no effects of maternal gestational weight gain on body fat distribution were found.

The effect of exposure to a diabetic intrauterine environment on child's adiposity has raised some controversy. A systematic review has yielded inconclusive results, but overall the associations were not statistically significant in 8 studies from a total of 12 included studies [5]. Additionally, a meta-analysis has reported an association between maternal diabetes and increased offspring BMI that was no longer significant after adjustment for maternal pre-pregnancy BMI [7]. In the present study, maternal diabetes tended to be positively associated with child's body fat quantity after taking maternal pre-pregnancy BMI into consideration. The intrauterine programming effects prevailed over the indirect effects through birthweight. Hyperglycemia is thought to induce excessive appetite postnatally [23], which might explain these results. Few studies have addressed the effect of maternal diabetes on child's body fat distribution, reporting, on one hand, a positive association with trunk fat from dual energy x-ray absorptiometry (DXA) [24] but, on the other hand, no association with visceral or subcutaneous abdominal fat obtained by magnetic resonance imaging [25]. In this study, no effects of maternal diabetes on body fat distribution were found.

A previous meta-analysis has suggested that maternal prenatal smoking leads to offspring obesity [4]. Thus far, some studies have reported positive associations whereas other studies have reported no associations between maternal prenatal smoking and child's central fat [26,27]. Our study showed that maternal smoking during pregnancy was associated with higher body fat quantity and a central fat distribution in childhood. These effects were mainly through intrauterine programming. The mechanisms by which maternal prenatal smoking may program child's adiposity involve an effect of cigarette smoke constituents, such as nicotine, which readily cross the placenta towards the fetus leading to permanent changes in the regulation of food intake and energy expenditure, such as increased appetite and decreased mobilization of fat from adipose tissue later in life [28,29]. Maternal smoking during pregnancy may also mimic fetal under-nutrition by reducing blood supply to the fetus via the vasoconstrictive effects of nicotine [2] or by reducing the mother's food intake [30]. Fetal under-nutrition has been associated with a reduced storage capacity of adipocytes, which may favor visceral fat deposition in the presence of a positive energy balance postnatally [31]. This could be one of the mechanisms involved in the higher accumulation of central fat in offspring of smoking mothers in our study. Moreover, fetal under-nutrition has been associated with reduced levels or a reduced effect of leptin during the postnatal period, thereby inducing excessive appetite [32]. A process favoring a better metabolic efficiency might also occur to enable 
fetal survival in a limited energy environment, which might lead to an excessive fat storage when enough food is available [1]. Even though weak, indirect effects through birthweight of maternal prenatal smoking on child's fat quantity and distribution were also observed in our study. Maternal smoking during pregnancy seems to lead to a child with low birthweight that subsequently maintains this low weight and tends to have a central fat distribution at 7 years old. Smoking delays fetus growth due to the high levels of carboxyhemoglobin and other toxic substances in blood, the vasoconstrictive effects of nicotine and fetal under-nutrition [2,3]. The maintenance of a low weight during childhood by low birth weight newborns could be explained, at least in part, by parental child-feeding practices, although this was not addressed in our study. However, we have previously shown in 4- and 7-year-old children of the Generation XXI birth cohort that a lower BMI leads to a greater parental use of pressure to eat that subsequently leads to a lower BMI [33]. Yet unknown mechanisms could be involved in the association of birthweight with body fat distribution.

Some limitations and strengths should be considered. Pre-pregnancy weight and final pre-delivery weight were both self-reported. Overall, weight tends to be underreported by women [34], which might have led to an underestimation of pre-pregnancy BMI. The attendance of prenatal visits could have made pregnant women more aware of their weight status, minimizing the error of selfreported final pre-delivery weight. Similarly, data on diabetes mellitus relied on self-reporting. If misclassification of women occurred, the association between maternal diabetes and offspring adiposity could be attenuated. Moreover, treatment of diabetes (based on diet or insulin), by contributing to a better glycemic control, may reduce the risk of long-term adverse outcomes for the offspring, thus complicating the detection of associations in observational studies like ours. Information on maternal smoking habits during pregnancy also relied on self-reporting, without biochemical validation, which may result in misclassification due to a reluctance to disclose a known adverse pregnancy behavior. However, any underreporting would likely bias results towards the null, underestimating the associations. We measured fat mass at 7 years old using BIA which is a valid method to assess whole body composition [35]. We relied on anthropometrics to assess body fat distribution which might have greater measurement error, and be less accurate but on the other hand be easier and cheaper to obtain in large epidemiological studies as compared to imaging techniques of body composition [36]. In this study, we considered that the direct effects reflected the intrauterine programming effects. We tried to perform a comprehensive analysis, by including the most important paths described to date, and by testing several covariates as confounders. However, we cannot exclude the possibility of existing other paths not considered in this study and also residual confounding by lifestyles or genetics that might have biased our estimates for the intrauterine programming effects. This seems unlikely and the bias might be only limited. The major strength of this study is the comprehensive assessment of the effects of prenatal exposures, by quantifying their direct and indirect effects, on uncorrelated and more robust measures of fat quantity and distribution in a large population-based sample of 7-yearold children.

\section{Conclusion}

This study showed that the effects of maternal weight gain, diabetes and smoking during pregnancy on body fat quantity of 7-year-old children seem to be mainly through intrauterine programming. Maternal smoking during pregnancy also showed a positive direct effect on body fat distribution of children. Considering the lasting and lifelong effects of intrauterine programming, primary prevention strategies for obesity, as early as during prenatal care, are of utmost importance. Although the indirect effects through birthweight seem to be weak, this study reinforces the need for careful interpretation of findings adjusted and not adjusted for birthweight in studies addressing the associations between prenatal exposures and adiposity in later life.

\section{Funding}

Generation XXI was funded by Programa Operacional de Saúde - Saúde XXI, Quadro Comunitário de Apoio III and Administração Regional de Saúde Norte (Regional Department of Ministry of Health). It has support through FEDER from the Operational Programme Factors of Competitiveness - COMPETE and through national funding from the Foundation for Science and Technology - FCT (Portuguese Ministry of Education and Science) within the project PTDC/SAU-EPI/121532/2010 (FCOMP-01-0124FEDER-021177) and from the Calouste Gulbenkian Foundation. Susana Santos received a grant from the Foundation for Science and Technology (SFRH/BD/81123/2011).

\section{Conflict of interest}

The authors have no conflicts of interest relevant to this article to disclose.

\section{Acknowledgments}

The authors gratefully acknowledge the families enrolled in Generation XXI for their kindness, all members of the research team for their enthusiasm and perseverance and the participating hospitals and their staff for their help and support.

The authors acknowledge the support from the Foundation for Science and Technology, the Caloust Gulbenkian Foundation and the Epidemiology Research Unit (EPI-Unit: UID-DTP/04750/2013). 


\section{References}

[1] Tounian P. Programming towards childhood obesity. Ann Nutr Metab 2011;58(Suppl. 2):30-41.

[2] Tabacchi G, Giammanco S, La Guardia M, Giammanco M. A review of the literature and a new classification of the early determinants of childhood obesity: from pregnancy to the first years of life. Nutr Res 2007;27:587-604.

[3] Fall CH. Evidence for the intra-uterine programming of adiposity in later life. Ann Hum Biol 2011;38:410-28.

[4] Ino T. Maternal smoking during pregnancy and offspring obesity: meta-analysis. Pediatr Int 2010;52:94-9.

[5] Kim SY, England JL, Sharma JA, Njoroge T. Gestational diabetes mellitus and risk of childhood overweight and obesity in offspring: a systematic review. Exp Diabetes Res 2011;2011: 541308.

[6] Mamun AA, Mannan M, Doi SA. Gestational weight gain in relation to offspring obesity over the life course: a systematic review and bias-adjusted meta-analysis. Obes Rev 2014;15:338-47.

[7] Philipps LH, Santhakumaran S, Gale C, Prior E, Logan KM, Hyde MJ, et al. The diabetic pregnancy and offspring BMI in childhood: a systematic review and meta-analysis. Diabetologia 2011;54: 1957-66.

[8] Richiardi L, Bellocco R, Zugna D. Mediation analysis in epidemiology: methods, interpretation and bias. Int J Epidemiol 2013;42: 1511-9.

[9] Basterfield L, Pearce MS, Adamson AJ, Reilly JK, Parkinson KN, Reilly JJ, et al. Effect of choice of outcome measure on studies of the etiology of obesity in children. Ann Epidemiol 2012;22: 888-91.

[10] Larsen PS, Kamper-Jorgensen M, Adamson A, Barros H, Bonde JP, Brescianini S, et al. Pregnancy and birth cohort resources in Europe: a large opportunity for aetiological child health research. Paediatr Perinat Epidemiol 2013;27:393-414.

[11] Husted JA, Cook RJ, Farewell VT, Gladman DD. Methods for assessing responsiveness: a critical review and recommendations. J Clin Epidemiol 2000;53:459-68.

[12] Kramer MS, Platt RW, Wen SW, Joseph KS, Allen A, Abrahamowicz M, et al. A new and improved population-based Canadian reference for birth weight for gestational age. Pediatrics 2001;108:E35.

[13] Schaefer F, Georgi M, Zieger A, Scharer K. Usefulness of bioelectric impedance and skinfold measurements in predicting fat-free mass derived from total body potassium in children. Pediatr Res 1994; 35:617-24.

[14] VanItallie TB, Yang MU, Heymsfield SB, Funk RC, Boileau RA Height-normalized indices of the body's fat-free mass and fat mass: potentially useful indicators of nutritional status. Am J Clin Nutr 1990;52:953-9.

[15] Stage FK, Carter HC, Nora A. Path analysis: an introduction and analysis of a decade of research. J Educ Res 2004;98:5-13.

[16] Hu L, Bentler PM. Cutoff criteria for fit indexes in covariance structure analysis: conventional criteria versus new alternatives. Struct Equ Model 1999;6:1-55.

[17] Muhlhausler B, Smith SR. Early-life origins of metabolic dysfunction: role of the adipocyte. Trends Endocrinol Metab 2009;20: $51-7$.

[18] Morris MJ, Chen H. Established maternal obesity in the rat reprograms hypothalamic appetite regulators and leptin signaling at birth. Int J Obes (Lond) 2009;33:115-22.

[19] Fraser A, Tilling K, Macdonald-Wallis C, Sattar N, Brion MJ, Benfield L, et al. Association of maternal weight gain in pregnancy with offspring obesity and metabolic and vascular traits in childhood. Circulation 2010;121:2557-64.

[20] Dello Russo M, Ahrens W, De Vriendt T, Marild S, Molnar D, Moreno LA, et al. Gestational weight gain and adiposity, fat distribution, metabolic profile, and blood pressure in offspring: the IDEFICS project. Int J Obes (Lond) 2013;37:914-9.

[21] Ensenauer R, Chmitorz A, Riedel C, Fenske N, Hauner H, NennstielRatzel U, et al. Effects of suboptimal or excessive gestational weight gain on childhood overweight and abdominal adiposity: results from a retrospective cohort study. Int J Obes (Lond) 2013; $37: 505-12$.

[22] Katzmarzyk PT, Bouchard C. Where is the beef? Waist circumference is more highly correlated with BMI and total body fat than with abdominal visceral fat in children. Int J Obes (Lond) 2014;38:753-4.

[23] Franke K, Harder T, Aerts L, Melchior K, Fahrenkrog S, Rodekamp E, et al. 'Programming' of orexigenic and anorexigenic hypothalamic neurons in offspring of treated and untreated diabetic mother rats. Brain Res 2005;1031:276-83.

[24] Chandler-Laney PC, Bush NC, Granger WM, Rouse DJ, Mancuso MS, Gower BA. Overweight status and intrauterine exposure to gestational diabetes are associated with children's metabolic health. Pediatr Obes 2012; 7:44-52.

[25] Crume TL, Ogden L, West NA, Vehik KS, Scherzinger A, Daniels S, et al. Association of exposure to diabetes in utero with adiposity and fat distribution in a multiethnic population of youth: the Exploring Perinatal Outcomes among Children (EPOCH) Study. Diabetologia 2011;54:87-92.

[26] Durmus B, Ay L, Hokken-Koelega AC, Raat H, Hofman A, Steegers EA, et al. Maternal smoking during pregnancy and subcutaneous fat mass in early childhood. The Generation R Study. Eur J Epidemiol 2011;26:295-304.

[27] Durmus B, Heppe DH, Taal HR, Manniesing R, Raat H, Hofman A, et al. Parental smoking during pregnancy and total and abdominal fat distribution in school-age children: the Generation R Study. Int J Obes (Lond) 2014;38:966-72.

[28] Jauniaux E, Burton GJ. Morphological and biological effects of maternal exposure to tobacco smoke on the feto-placental unit. Early Hum Dev 2007;83:699-706.

[29] Levin ED. Fetal nicotinic overload, blunted sympathetic responsivity, and obesity. Birth Defects Res Part A Clin Mol Teratol 2005; 73:481-4.

[30] Jo YH, Talmage DA, Role LW. Nicotinic receptor-mediated effects on appetite and food intake. J Neurobiol 2002;53:618-32.

[31] Danforth Jr E. Failure of adipocyte differentiation causes type II diabetes mellitus? Nat Genet 2000;26:13.

[32] Yura S, Itoh H, Sagawa N, Yamamoto H, Masuzaki H, Nakao K, et al. Role of premature leptin surge in obesity resulting from intrauterine undernutrition. Cell Metab 2005;1:371-8.

[33] Afonso L, Lopes C, Severo M, Santos S, Real H, Durao C, et al. Bidirectional association between parental child-feeding practices and body mass index at 4 and 7 y of age. Am J Clin Nutr 2016;103: $861-7$.

[34] Brunner Huber LR. Validity of self-reported height and weight in women of reproductive age. Matern Child Health J 2007;11: $137-44$.

[35] Luque V, Closa-Monasterolo R, Rubio-Torrents C, ZaragozaJordana M, Ferre N, Gispert-Llaurado M, et al. Bioimpedance in 7year-old children: validation by dual X-ray absorptiometry - part 1: assessment of whole body composition. Ann Nutr Metab 2014; 64:113-21.

[36] Wells JC, Fewtrell MS. Measuring body composition. Arch Dis Child 2006;91:612-7. 